\title{
Avatar-Mediated Communication in Social VR: An In-depth Exploration of Older Adult Interaction in an Emerging Communication Platform
}

\author{
Steven Baker \\ Griffith University \\ Southport, QLD, Australia \\ steven.baker@griffith.edu.au \\ Ryan M. Kelly \\ University of Melbourne \\ Melbourne, VIC, Australia \\ ryan.kelly@unimelb.edu.au \\ Briony Dow \\ National Ageing Research Institute \\ Melbourne, VIC, Australia \\ b.dow@nari.edu.au
}

\author{
Jenny Waycott \\ University of Melbourne \\ Melbourne, VIC, Australia \\ jwaycott@unimelb.edu.au
}

Anthony Jones

University of Melbourne

Melbourne, VIC, Australia

a.jones@unimelb.edu.au

Frances Batchelor

National Ageing Research Institute

Melbourne, VIC, Australia

f.batchelor@nari.edu.au

Frank Vetere

University of Melbourne

Melbourne, VIC, Australia

f.vetere@unimelb.edu.au

\author{
Romina Carrasco \\ University of Melbourne \\ Melbourne, VIC, Australia \\ romina.carrasco@unimelb.edu.au \\ Jack Lilley \\ University of Melbourne \\ Melbourne, VIC, Australia \\ jl@vic.chariot.net.au \\ Thuong Hoang \\ Deakin University \\ Geelong, VIC, Australia \\ thuong.hoang@deakin.edu.au
}

\begin{abstract}
While HCI researchers have begun designing personalised VR experiences for older adults, there has been limited research examining the use of social VR - where users interact via avatars in a virtual environment. Avatar-mediated communication (AMC) is a crucial component of the social VR experience, but older users' experience with AMC is poorly understood. We conducted a five-month study with 16 older adults evaluating a co-designed social VR prototype. Results show that AMC in social VR was seen as medium that supported introverted users to express themselves and was viewed as offering advantages when discussing sensitive topics. Our study provides new insights into how older adults view AMC in social VR as a communication medium and we contribute six design reflections, based on our results, that highlight the steps that can be taken to ensure that AMC in social VR can meet the communication needs of older users.
\end{abstract}

\section{CCS CONCEPTS}

- Human-centered computing $\rightarrow$ Empirical studies in $\mathrm{HCI}$.

\section{KEYWORDS}

Avatars; Older adults; Social VR

CHI '21, May 8-13, 2021, Yokohama, Japan

(c) 2021 Copyright held by the owner/author(s). Publication rights licensed to ACM.

This is the author's version of the work. It is posted here for your personal use. Not for redistribution. The definitive Version of Record was published in CHI Conference on Human Factors in Computing Systems (CHI '21), May 8-13, 2021, Yokohama, Japan, https://doi.org/10.1145/3411764.3445752.

\begin{abstract}
ACM Reference Format:
Steven Baker, Jenny Waycott, Romina Carrasco, Ryan M. Kelly, Anthony Jones, Jack Lilley, Briony Dow, Frances Batchelor, Thuong Hoang, and Frank Vetere. 2021. Avatar-Mediated Communication in Social VR: An In-depth Exploration of Older Adult Interaction in an Emerging Communication Platform. In CHI Conference on Human Factors in Computing Systems (CHI '21), May 8-13, 2021, Yokohama, Japan. ACM, New York, NY, USA, 13 pages. https://doi.org/10.1145/3411764.3445752
\end{abstract}

\section{INTRODUCTION}

In recent decades, the percentage of older adults as a proportion of the population has increased in many regions across the globe, leading to a renewed focus on research into the ways that computermediated communication might be used to help older people to stay socially connected [4]. The worldwide COVID-19 pandemic has only served to reinforce the importance of technology as a communication medium, especially for older adults [12, 23, 34]. It has also brought increased awareness of the dangers of social isolation and the importance of maintaining contact with our friends and loved ones when we are unable to meet face-to-face [34].

In this paper, we report on a five-month user study that investigated the benefits of avatar-mediated communication in social virtual reality (hereafter, AMC in social VR), an emerging social technology that has potential to respond to the needs of older adults [5]. Avatar-mediated communication combines two of humanity's most enduring obsessions: the ability to communicate with other people over distance (e.g. the telegraph, the telephone and the internet), and the desire to build human-like machines that are imbued with aspects of our personalities (e.g. automatons, puppets and robots). AMC in social VR can be distinguished from 
other forms of computer-mediated communication in two primary ways. First, users communicate via embodied avatars - digital representations of a human user that are controlled via a natural user interface [25]. Second, social VR - which utilises a head-mounted display (HMD) and body-tracking sensors that allow users to control their avatars via natural body movements - has the unique property of being able to transport the user to a fully immersive virtual world [42] and give them agency over their embodied avatar. When combined, these attributes offer potential advantages over other forms of computer-mediated communication. For example, users can convey both verbal and non-verbal cues via their avatars while maintaining a level of anonymity and privacy [1, 39]. AMC in social VR also allows users to feel co-present in virtual space, collapsing the physical and psychological distance that is often experienced when using other communication platforms [1]. Lastly, it is possible to design social VR environments that offer rich experiences akin to those people enjoy face-to-face, such as photo sharing [21], or the exchange of objects among virtually co-located users [2].

To date, much of the research into older adults' use of AMC in virtual worlds has been limited to screen-based applications such as Second Life or IMVU [13, 30, 38-40]. Older adults' evaluations of these systems have identified that although AMC in virtual worlds could be useful for social interaction, issues such as a lack of nonverbal cues were perceived to limit the usefulness of 3D avatars for communication [13, 30, 38]. Baker and colleagues conducted a short-term evaluation of a prototype social VR system that allowed groups of older adults to meet and communicate on a virtual island [3]. While the participants in their study agreed that AMC in social VR had potential as a communication tool, body tracking errors and a lack of behavioural anthropomorphism - an avatar's ability to act in a human-like manner - acted as significant barriers to communication. In the system described in this paper, we address these challenges by incorporating measures aimed at improving the sense of presence in the virtual environment and strengthening the connection between the older user and their avatar using features recommended by Baker and colleagues [3]. We also responded to prior work that has called for longer term evaluations of VR systems aimed at older users [43], by using a participatory methodology that supported older adults to play a more central role in evaluating the prototype and adopting an expanded study timeline so that our participants would have time to fully evaluate the prototype. The user study also sought to create a more realistic communication environment by engaging two groups of older adults who were geographically dispersed over a distance of $150 \mathrm{~km}$ (93 miles). By adopting these measures, we aimed to better understand how older adults experience and view AMC in social VR. Sixteen adults aged between 70 and 81 took part in a five-month user study that evaluated a social VR prototype they had co-designed in a previous series of participatory workshops. In this paper, we draw on data collected during the user study to respond to the question: How can older adult experiences of AMC in social VR inform the design of social VR systems aimed at older users?

Our results demonstrate how AMC in social VR can positively impact on the ability of shy and introverted users to contribute to group discussion, which is significant given that older adults with these personality traits are particularly susceptible to the adverse impacts of social isolation [27]. Moreover, our results show that participants found AMC in social VR to have benefits over face-to-face communication when discussing sensitive information. Based on these findings, we contribute suggestions for how future social VR systems might be used as tools to engage older adults experiencing depression and social isolation. Our participants also articulated how some of the unique characteristics of avatars might be used to enhance communication in certain circumstances, and how AMC in social VR addresses problems associated with divided attention that impact on videoconferencing platforms. Finally, as our prototype implemented recommendations from earlier work by Baker and colleagues [3] aimed at increasing the behavioural anthropomorphism of avatars in social VR, we examine our participants' reactions to these and consider how these can be used to further improve the design of future social VR systems aimed at older users. In responding to our research question, we contribute six design reflections that speak to how the insights gained from the user study can be used to inform future design and research into older adults use of AMC in social VR.

\section{RELATED WORK}

Our research builds on related work that has evaluated avatarmediated communication in both screen-based and fully immersive virtual worlds, and research into the use of VR by older adults.

\subsection{AMC in Social Virtual Worlds and Social VR by Older Adults}

Relatively little research has focused on AMC in social VR by older users. However, previous work has studied the use of social virtual worlds such as Second Life by older adults [30]. These worlds are similar to social VR in that they use digital avatars to represent the user in a shared 3D virtual environment, but do not usually involve the use of HMDs, hand controllers or body tracking technologies.

Siriaraya and colleagues have completed numerous studies investigating older adult communication in both commercial and bespoke social virtual worlds [36-40]. Their work suggests that a major advantage of social virtual worlds over other communication technologies lies in its potential ability to provide older users with an augmented sense of realism, creating greater levels of presence - both within the virtual environment and with others sharing the virtual world [38-40]. They note that both social and physical presence are factors that correlate with "overall satisfaction in the social experience and quality of communication" for older users [40, p.288]. In one study that involved interviewing 15 older users (aged 55 to 65) of Second Life and IMVU, Siriaraya and colleagues [39] report that AMC provided a level of anonymity that allowed the older users to blend into communities that were dominated by younger users [39]. However, in more recent work with 38 older users (aged between 55 and 82) who interacted in a screen-based virtual shopping activity, results indicated that older adults "found 3D avatars to be of limited use to their social interactions" [38, p. 114]. In considering these results, Siriaraya and Ang propose a number of "steps that could be taken to improve avatar-mediated communication for older users" [38, p.114]. These include providing 
separate $2 \mathrm{D}$ photographs to identify the users to improve transparency, and incorporating gesture based sensors to facilitate the transmission of non-verbal cues [38].

O'Brien, Smith and Beck [26] conducted a study with 36 older adults exploring social engagement in Second Life. They also found that some participants believed that an advantage of AMC in Second Life was that communicating via an avatar provided a level of anonymity that they enjoyed. However, a number of challenges hampered the quality of communication. Several of the less outgoing participants felt that communication in Second Life was only going to suit those who were "really outgoing" [26, p.175]. Others felt afraid when trying to communicate with strangers. One interviewee also felt the "lack of real life connection was a barrier" to building meaningful relationships [26, p.175]. They compared Second Life to communicating in Facebook and commented that the lack of information about a communication partner's location and a lack of social context via the ability to view photographs etc., meant that they were "unable to bond" [26, p.176].

The above related work suggests that while older adults see AMC in social virtual worlds as having the potential to support meaningful communication, there were mixed results in terms of the actual experience of AMC. It is unclear whether these findings apply to the use of AMC in social VR.

We are aware of only one study that has considered AMC in social VR. Baker and colleagues [3] report on a study that saw 25 older adults aged between 70 and 81 engage in a social VR prototype that allowed groups of four people to visit a virtual island and engage in conversation. Results from this study showed that while the participants saw great potential for AMC in social VR to be used as a tool to address social isolation, several factors impacted on their ability to feel socially engaged during the study. In particular, they highlighted factors related to behavioural anthropomorphism, which refers to their embodied avatars' ability to speak, move, and act in a human-like manner, and other factors relating to the way the embodied avatars' non-verbal gestures were mapped in the prototype. In subsequent participatory design work, Baker and colleagues partnered with the same participants to produce a design concept for a social VR application that would respond to participants' communication needs [5].

\section{$2.2 \quad$ VR and Older Adults}

While we have noted the lack of research relating to older adults and social VR, an increasing body of literature has examined how older users might utilise VR systems to support healthy ageing. This work has covered a broad range of interventions including the use of VR as an aid to balance rehabilitation [18]; improving strength and mobility by using VR gaming software [20,41]; using VR as a tool to aid memory function $[17,19]$; and exploring how VR can be used to address depressive symptoms in older users $[6,35]$. Roberts and colleagues [32] conducted a qualitative evaluation of an immersive VR system with 41 older residents of a retirement community. Their participants identified four types of VR content they felt would be valuable to older users: travel; education; reminiscence; and self-care/therapeutic [32]. Similar preferences have been noted in other studies that have asked older adults about the types of VR experiences that they would like to see offered to older users [5]. The participants in the Roberts study expressed a "high level of interest in VR for the future" but criticised the system used in the study as they felt it made them "like a passive observer, rather than an active participant" saying they felt "inadequate social connectedness" [32, p.5-7]. This supports the view that older adults are interested in multi-user VR systems that can support social interaction.

In a recent systematic review of the use of VR with older adults, Tuena and colleagues [43, p. 1] note that while many VR systems were rated as "usable and feasible", most of the reviewed literature used less-immersive (i.e. screen-based) systems. They call for more long-term studies to evaluate the use of VR with older adults using "step-by-step" user-centred design incorporating "pretesting" and "prototype development" in order to ensure that VR systems respond to the usability needs of older users [43, p.15].

The related work above speaks to the potential for AMC in social VR to be an engaging communication medium for older adults. However, there are clear gaps in our knowledge about how best to design social VR systems to support AMC. The study reported in this paper seeks to respond to several of the gaps identified in the related work. We respond to Tuena and colleagues' calls for longer term studies that evaluate VR with older adults by conducting what we believe to be the first such study of a social VR prototype by older users. Importantly, our study builds on earlier participatory work with the same participants that included the co-design of the social VR prototype used in the study. Recognising the importance of presence and behavioural anthropomorphism to effective AMC, our study also incorporates design recommendations from prior work in order to advance our knowledge of how these factors impact on older adults' AMC.

\section{PARTICIPATORY METHODOLOGY AND CONTEXT}

Our study is grounded in a three-year research program that has explored how social VR might be designed to support the needs of older adults. While the present paper focuses on the five-month user study in which we evaluated older adults' experiences with AMC in social VR, the study was preceded by in-depth participatory research with the participant group. In this section, we outline the methodological foundations of the project - which utilised participatory action research (PAR) - and briefly describe the three participatory cycles of research that preceded the user study.

PAR adopts a critical realist stance that seeks to reveal the "causal mechanisms" that underpin our experience of the world [24, p.109]. The active participation and empowerment of the participants are central to this approach $[16,29]$. The methodology does not consider this as contamination or bias, but rather as "an inevitable part of the social construction of scientific knowledge" [16, p. 7]. PAR adopts an approach to rigor that is centred on the notion of trustworthiness [16], a key aspect of which is involving participants in all aspects of the research process. As Hayes notes in her examination of the use of action research in HCI, unlike other participatory approaches, the inclusion of participants "does not end with the implementation of the research nor with the analysis of results. Rather, action research explicitly requires writing with engaged partners" [16, p. 11]. 
Prior to taking part in the user study reported here, the participants had been involved in two previous action research cycles. In the first cycle, the participants took part in a series of workshops that were designed to familiarise them with VR and the use of avatars. This culminated in a workshop where they trialled a basic social VR prototype that allowed them to control embodied avatars via a Microsoft Kinect sensor [3]. In the second cycle, a subset of the same group of participants were involved in the participatory design of a social VR application concept [5] that was used as the basis for the prototype that was developed for the user study [2].

The PAR process continued to inform the user study reported in this paper. The participants were invited to try early builds of the prototype before the study in order to identify areas where improvements were needed. They also co-designed avatars with the third author to ensure the avatars were personalised to meet their needs, and were invited to attend research meetings that occurred between user study sessions. These meetings allowed them to discuss any issues they had in the previous sessions. Improvements to the prototype were prioritised based on their comments.

In a continuation of this commitment to ongoing participation and trustworthiness, we invited two of the participants to be coauthors on this paper (fifth and sixth authors). This follows the recommendations of Hayes [16] and gives the participants an active voice in how the research is presented.

\section{METHOD}

\subsection{Social VR Prototype}

The data we draw on in this paper was collected as part of an evaluation of School Days, a social VR prototype that enabled participants to meet in a virtual environment to reminisce about school experiences. The prototype was built using Unity3D (Version 2017.3.0f3) and allowed up to four users to meet simultaneously in a virtual environment that was modelled on a school classroom. We developed the prototype to incorporate a facilitator as one of the four users who would be present in the virtual environment during each of the user study sessions. This was based on a design recommendation from the participants who felt that AMC would be aided by having an 'expert' present to provide in situ guidance and support. The first author, who has a background as a social worker specialising in work with older adults, took on the facilitator role throughout the study.

We designed School Days for use with a head-mounted display. Figure 1 shows one of our participants using the prototype during a social VR session. The setup for each user consisted of an Oculus Rift VR system tethered to a gaming PC and monitor. Two Oculus Touch hand controllers and two Rift room tracking sensors were used to allow fine-grained control over the avatar's hand and body movements. This was a design response to participants' criticism of an earlier prototype system that had used a Microsoft Kinect sensor to map body movements. Participants were able to communicate via a high-fidelity audio feed incorporated into the prototype system. The prototype was designed to be used in a seated position to reduce the risk of simulator sickness [18] and to support participants who had mobility constraints.

The prototype contained two virtual environments, The Hall and The Classroom. The Hall was used as an onboarding environment

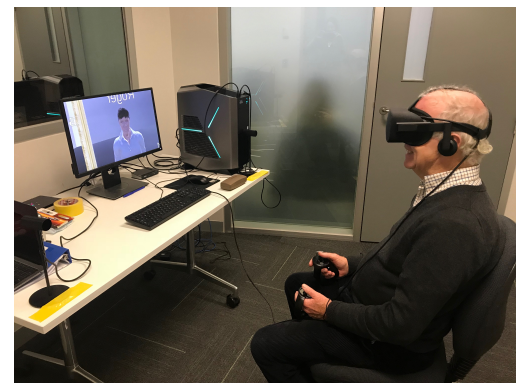

Figure 1: Samuel using the Oculus system during a social VR session.

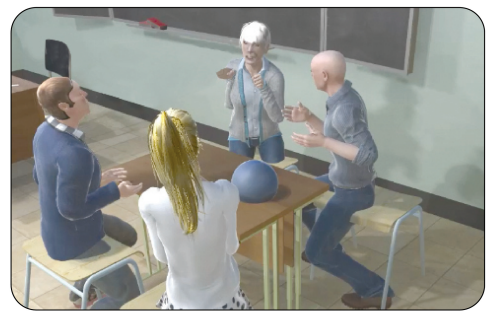

Figure 2: Participants hold a conversation in The Classroom.

and consisted of a plain room with a table and four chairs. The Hall was the first place that users entered and was the place where introductions took place when users joined a session.

The Classroom was the main site where AMC took place and where participants spent the majority of each session. The Classroom was decorated to resemble a modern school classroom with desks and chairs, a blackboard and various classroom paraphernalia. Participants were seated around a desk at the 'head of the class' while in the environment. The virtual space was also furnished with a range of reminiscence scaffolding features that have been described in prior work [2]. Figure 2 shows participants engaged in conversation in The Classroom.

\subsection{Avatars and Expression Features}

When using School Days, participants were able to embody two bespoke avatars they had co-created in participatory design sessions led by the third author. We encouraged participants to design two avatars to give them the option to express themselves in different ways during the user study sessions. In this paper, we focus on the participants' general impressions of social interaction in AMC, as opposed to their reaction to the specifics of their own avatar designs.

The first avatar - which we referred to as the realistic avatar was designed to be a lifelike representation of the user. The second avatar - which we referred to as the participants' cartoon avatar was a free-form design that allowed them to express themselves in a more unique way. Figure 3 shows a selection of the avatars that were co-designed by participants. When in The Hall, participants were able to use their hand controllers to pull up a virtual mirror that 


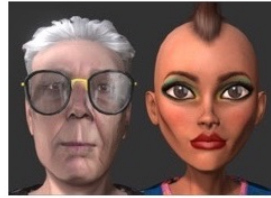

Cleo

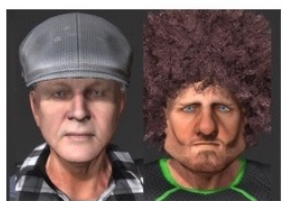

Carl

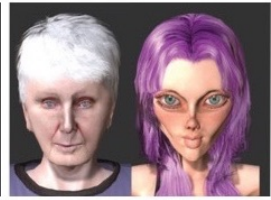

Holly

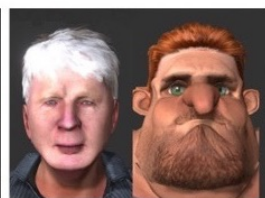

Herb

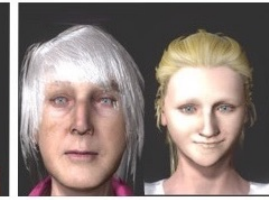

Amy

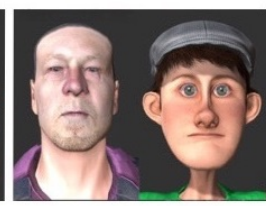

Harry
Figure 3: Pairs of avatars created by six of our participants. Each pair shows the participant's realistic (left) and cartoon avatar (right) along with their pseudonym from Table 1.

allowed them to choose an avatar for the session and rehearse how it behaved when they were talking and moving. Participants were able to see one another's real name floating above their avatar's head, so that they could identify who was who easily.

Based on prior work and recommendations from earlier research cycles in the project, School Days incorporated specific avatar expression features designed to respond to our participants' requests for their avatars to have higher levels of behavioural anthropomorphism [3,25]. This included adding facial and body movements (via the Final IK plugin by Root Motion ${ }^{1}$ ), and lip movements (via the OVRLipSync plugin by Oculus ${ }^{2}$ ). These features are described in further detail in our results section.

\subsection{Participants}

We recruited a total of 16 older adult participants for the user study. All had been involved in earlier stages of the research project and had initially been recruited via advertisements disseminated in public forums frequented by older adults and through posters that had been circulated in public spaces such as libraries and community noticeboards. In order to be included in the project, participants were required to be over the age of 70, self-identify as able to speak English to a reasonable standard and have an interest in technology. At the commencement of the project, each participant chose a pseudonym (listed in Table 1) and these were used throughout the project.

Five participants identified as female and eleven as male. All were aged between 70 and 81 at the time of the study. Participants were geographically distributed across two research sites. Five (4 male, 1 female) were located in a regional location and eleven (7 male, 4 female) were located in a major metropolitan centre $150 \mathrm{~km}$ (93 miles) from the regional participants. While each group had been involved in the earlier stages of the project, these research activities had been completed separately so that the regional and

\footnotetext{
${ }^{1} \mathrm{http}: / /$ www.root-motion.com/final-ik.html

${ }^{2}$ https://developer.oculus.com/downloads/package/oculus-lipsync-unity/
}

metropolitan groups would 'meet' for the first time in social VR during the user study.

All participants were either retired or semi-retired and lived independently in the community, except for one who lived in residential aged care. Eight participants reported specific age-related health issues. Two female participants used mobility aids when walking, four male participants had hearing impairments that required the use of hearing aids and one participant reported a mental health condition that - at times - impacted on their ability to socialise. In addition to these issues, one participant was living with Parkinson's disease and they required medications to assist with communication and movement. These health issues were taken into account when designing our research and risk management procedures, but none of these issues had severe impacts on the participants' ability to communicate when using the prototype.

\subsection{Procedure}

We conducted a total of 26 social VR sessions during the five-month study. Each session lasted for 27-56 minutes (average $=44$ minutes). All sessions involved two or three participants and the facilitator. Sessions ran to a fortnightly schedule, during which two or three sessions took place over a single day during the scheduled week. This schedule was developed to provide a regular routine for our participants while providing ample opportunities to enrol in a social VR session. Participants were able to self-enrol in as many sessions as they wished during the study, but did not have knowledge of which other participants would be in the same session. In the initial sessions, some participants were meeting for the first time because they were located at either the regional or metropolitan site. Participants then became more familiar with each other as the study progressed, primarily because they had met in previous social VR sessions. This meant our study captured a mixture of interactions between strangers getting to know one another, and conversations between people who were already acquainted.

As shown in Table 1, participation rates ranged from one to seven sessions. The participants were generally keen to try the prototype, given that they had co-designed it in an earlier phase of the research [5] and so we did not need to encourage them to sign up for the sessions. The differing rates of participation shown in Table 1 were due to factors such as participants' holiday commitments, caring responsibilities or scheduling clashes with other activities.

User study sessions were conducted across two university campuses. These sites were chosen as they allowed for the control of variables such as network speed and access, while also being convenient places for the participants to visit. During the sessions, participants were dispersed across various physical locations (typically a quiet research laboratory or faculty office) at either site. Members of the research team were located beside each participant at all times to provide instructions and assistance while the sessions were taking place. The researchers also collected observation data during the sessions and surveyed each participant's experience once the session had concluded.

The procedure for each session was as follows. Sessions began with each participant sitting on a chair, reviewing the process for manipulating their avatar via the hand controllers, and then being assisted to put on the VR gear (HMD and Oculus Touch controllers). 
Table 1: User study participant and session information.

\begin{tabular}{lrr}
\hline Participant Pseudonym & Number of Social VR Sessions & Location \\
\hline Wes & 4 & Regional \\
Gordon & 4 & Regional \\
Klaus & 5 & Regional \\
Len & 3 & Regional \\
Tina & 3 & Regional \\
Herbert & 5 & Metropolitan \\
Samuel & 4 & Metropolitan \\
Yulia & 4 & Metropolitan \\
Carl & 2 & Metropolitan \\
Bernard & 7 & Metropolitan \\
Evan & 1 & Metropolitan \\
Herb & 5 & Metropolitan \\
Harry & 4 & Metropolitan \\
Cleo & 6 & Metropolitan \\
Holly & 5 & Metropolitan \\
Amy & 4 & Metropolitan \\
\hline Total & 66 & \\
\hline
\end{tabular}

The researcher then started the prototype using a laptop. Participants first appeared in The Hall and went through a process to get 'dressed' as their avatar. They then introduced themselves to the other avatars seated around the table before collectively deciding to enter The Classroom for extended periods of communication. The sessions did not have predefined themes or topics for discussion. Instead, participants used features within School Days to generate topics for discussion [2]. These features included 'conversation starters', which were questions generated by pressing a button on the virtual desk (e.g. "what school trips and excursions did you take?"); virtual 3D artefacts like photographs or trophies from their youth that participants could pass around the group; and 3D avacasts, which were virtual holograms that played pre-recorded stories from other older adults in the study [2]. Each of these features helped to stimulate conversation and encourage interactions between the participants.

At the conclusion of each session, the facilitator invited the participants to teleport back to The Hall, where they were able to say goodbye and end the social VR session. The accompanying member of the research team then helped the participant to remove the VR gear. Each participant immediately filled out an adapted version of Witmer and Singer's presence questionnaire [45] that aimed to capture their sense of having 'been there' in the virtual environment. Each participant then took part in a semi-structured interview to discuss experiences during the social VR session.

\subsection{Data Collection and Analysis}

The user study produced 66 interviews comprising 12 hours and 15 minutes of audio, and 18 hours and 26 minutes of screen-captured video of AMC in social VR. The majority of this video was recorded from the perspective of the facilitator's HMD, though in later sessions a feature was added to allow us to capture video from a dedicated virtual camera embedded in the virtual environment.
Members of the research team sitting beside the participants during the social VR sessions also took photos and short video recordings to capture notable interactions and points of interest. In total, 8 hours and 30 minutes of additional video footage was recorded. Research team members also took observational notes. This qualitative data was transcribed where necessary and then imported into NVivo (version 12) for further analysis.

A questionnaire was administered at the conclusion of each user study session (66 responses in total). We asked VR presence questions that were adapted from an earlier questionnaire developed by Witmer and Singer [45]. Table 2 shows the questions and summaries of responses to the questionnaire. In this paper, we use the questionnaire data to provide descriptive statistics in support of Theme 4, which relates to emotions and non-verbal cues in AMC.

We conducted a thematic analysis of the video, photos and interview data following Braun and Clarke's six phase approach [8, 9]. Having transcribed, read and re-read the data (Phase 1), we adopted an inductive approach to coding the data (Phase 2), resulting in 101 codes. We began searching for themes (Phase 3), by collating the codes into potential thematic areas. Phases 4 through 6 involved refining the themes through thematic mapping, consolidation of codes and discussion among the authors. This resulted in four themes that we describe in the results section.

\section{RESULTS}

Thematic analysis of the user study data produced four themes that illustrate the impact that AMC in social VR had on the participants and their thoughts on its usefulness to older adults. The themes are complementary, in that they shed light on the individual ways in which our participants made sense of this type of communication, while also painting a picture of the richness of their collective experience.

\subsection{Theme 1. Because it's all virtual it throws inhibitions away: Comparing AMC with other forms of communication.}

Participants often reflected on the way the experience of AMC compared to other communication methods, particularly in the semi-structured interviews that took place immediately after each session. Our first theme reflects this rich seam of data, coalescing around two sub-themes. The first relates to comments from the participants about how their experience of AMC differed from faceto-face communication in real life, while the second details the participants' thoughts about how AMC compares to other forms of computer-mediated communication, specifically videoconferencing software such as FaceTime or Skype.

5.1.1 Comparing AMC to face-to-face communication. Understandably, our participants often reflected on how their experience of AMC compared to conversations that happen in real life. For some, such as Len, there was a clear preference for face-to-face communication, typified by a comment made towards the end of the user study sessions: "well my preference would be to meet face-to-face, very much so". In the early stages of the user study, Klaus commented that while the quality of the conversations in social VR "were good...like having a conversation if you were in the room with 
Table 2: Means and standard deviations for responses to the presence questionnaire (adapted from [45]). All ratings were given on a 1-5 Likert scale where $1=$ Strongly disagree and $5=$ Strongly agree.

\begin{tabular}{|c|c|c|}
\hline Question & Mean & S.D. \\
\hline $\begin{array}{l}\text { How quickly did the virtual environment respond to your ac- } \\
\text { tions? (movements, grabbing) }\end{array}$ & 3.74 & 0.95 \\
\hline $\begin{array}{l}\text { Were you engaged with the virtual environment in terms of } \\
\text { visual information? }\end{array}$ & 3.88 & 0.73 \\
\hline $\begin{array}{l}\text { Were you engaged with the virtual environment in terms of } \\
\text { audio information? }\end{array}$ & 3.98 & 0.90 \\
\hline $\begin{array}{l}\text { Were you engaged with the virtual environment in terms of } \\
\text { interaction with objects? }\end{array}$ & 3.76 & 0.82 \\
\hline $\begin{array}{l}\text { How much did your experiences in the virtual environment } \\
\text { seem consistent with your real-world experiences? }\end{array}$ & 3.50 & 0.89 \\
\hline
\end{tabular}

people", he felt the conversations were largely reliant on the quality of the other participants' voices, with avatars only serving as "something to concentrate on". For others, however, AMC offered unique communication benefits.

For Holly, a shy person who rarely spoke in group conversations except when being addressed directly, AMC in social VR offered an environment that was more conducive to social participation:

Well...if the four of us [had] been sitting around a table [in the real world], I would've felt a bit uncomfortable about talking. But because it's all virtual, it sort of comes freely; the talking...it throws inhibitions away or something.

Results from the thematic analysis also highlighted that many participants thought $\mathrm{AMC}$ provided specific benefits with respect to supporting a level of anonymity that they appreciated. Herbert felt that a benefit of AMC was that it "removes [conversation] from direct person-to-person interaction. You have an avatar in between.". He noted that "people are often better able to talk about abstractions and things removed from themselves." Yulia similarly described AMC as offering an environment where it was "easier to talk about...people in positive terms, without necessarily identifying them."

These comments are supported by our broader analysis of the user study data. Analysis of the video footage captured in the virtual environment revealed that participants frequently discussed highly personal - and often traumatic - experiences in the sessions, including childhood abuse, losing family members, and being sent to homes for 'destitute' children. Yulia described the impact of losing her sister when they were trapped in a bombed building in Europe during World War Two. In the subsequent interview, she reflected on the way AMC in social VR supported intimate sharing:

Well I think it's easier. I think it was a good interaction. I think it's easier to ask people fairly personal questions... via their avatar, rather than face-to-face, because you know that the avatar is VR and if the person doesn't really want to answer, then they don't have to, and they don't have to feel uncomfortable about not answering. Whereas, I think if you are speaking person-to-person, as we are at the moment, [and] you ask me a question that I didn't particularly want to answer, but felt compelled to answer, you could tell this from my face, whereas this way [via AMC] I can keep my feelings hidden from you, but answer your question to the best of my ability.

The above quote suggests that AMC in social VR may be able to play a role in supporting shy or introverted older adults, who may be uncomfortable in traditional face-to-face settings, to feel more empowered to participate in social activities. These results also support the view that AMC in social VR supports a level of anonymity that prior work has demonstrated as being valued by older users [26, 39]. However, our results extend these earlier findings by demonstrating how older adults found that AMC supported the discussion of highly personal experiences. Finally, the results suggest that many of our participants believed that AMC in social VR has advantages over face-to-face conversations when discussing sensitive topics.

5.1.2 Comparing AMC to prior experiences with videoconferencing software. Throughout the user study, participants made frequent comparisons between AMC in social VR and their experience of using videoconferencing software. Gordon felt that AMC had "a long way to go before it comes to the standard of something like FaceTime or Skype." Len expanded on this thought by providing some specific examples of the advantages he believed made videoconferencing software more appealing than AMC in social VR: "[with videoconferencing software] no headset [HMD] required. I turn on my phone [or] my iPad [and] talk to my granddaughter in Thailand... With FaceTime, you can push a button and she can show you the scenery around where she is... and I'm seeing her, I'm not seeing someone who looks like her."

Interestingly, however, Harry - who also reported using videoconferencing software regularly to talk with family who are geographically dispersed - felt AMC in a virtual world "would be a fantastic progression or enhancement for Skype." Harry felt it was precisely the fact that you would be located in a unique virtual environment that made this appealing. "You could pick [a virtual environment] and it [wouldn't] have to be the kitchen at home. It could be anywhere else, like a coffee shop...or Buenos Aires or something...You could choose the environment and chat to family who are anywhere...”. As the user study progressed, Klaus - who had initially seen limited use for AMC - changed his view and commented:

I've revised my opinion of avatars...The way it works with this [AMC in social VR] is probably the best sort of metaphor you could have for dealing with people who are a distance apart...[Using videoconferencing technology] you would need to represent [users] on some sort of screen, and the only way I can think of at the moment is if you [divided the screen into] four pictures... The system we are using is...I think, more natural...because you can have everybody in the same room...I think [AMC in social VR] has got enormous potential for getting - particularly people who you can't get out of where they're living - an aged care home or their own home, connecting.

Samuel, rather than highlighting the advantages of AMC in relation to space and place, saw AMC and social VR as offering a solution to the problem of divided attention. He recalled a recent 
video call with his son: "I was just talking to my son in Rotterdam on WhatsApp last night. He was obviously doing something else. I felt like saying 'look, give me your full attention!'. Samuel's comment highlights one of the potential unique advantages of AMC in social VR; because users access the social VR environment via a HMD, and their body movements are tracked in real time, they are fully immersed in that world. That is, the technology allows multiple, geographically dispersed users to meet in an immersive space that removes them from the everyday distractions that can hamper other forms of computer-mediated communication [46].

It is clear from these results that not all our participants saw AMC in social VR as comparing favourably to more mature forms of computer-mediated communication, such as teleconferencing software, though their comments do begin to shed light on specific design priorities that will need to be met if social VR is to become a mainstream communication platform, a topic to which we will return in our discussion.

\subsection{Theme 2. You can just reveal yourself as you feel comfortable: The impact of avatar appearance on AMC}

Thematic analysis revealed a range of reactions to the impact of avatar appearance on the communication that occurred in the social VR application. An aspect of AMC that many people commented on was the way that an avatar's appearance can be adapted to suit a particular communication context. Harry reflected that this ability to adapt an avatar's appearance could offer particular benefits for some older adults:

[It] could be really important to be an avatar [when] getting to know someone else. Because then it's just tipping your toe in the water and getting to know... rather than being all there immediately....and you can just reveal yourself as you feel comfortable.

Bernard suggested he would rather use a realistic avatar "If I was going to do communication with certain grandchildren.” In reflecting on his recent 50th wedding anniversary, however, he suggested that he might create an avatar that conveyed "what we looked like in 1967 when we were married" for that social context. Herb, while typically using his realistic avatar during the user study, felt that you might need "the cartoon one in a totally different environment, with a totally different subject to talk about... [one] more related to what you would like to be". Samuel, who chose to design a female character as his cartoon avatar, wondered if it would offer him the ability to 'better fit in' when having conversations with women. In reflecting on a conversation with a woman participant he commented, "if I'd changed my avatar to the female one, [would she] have talked differently while looking at a female image rather than a male one?" Amy chose to use a cartoon avatar in the prototype that was based on a photo of her as a twelve-year-old child. In reflecting on this choice she commented:

I just think it is fun to use, and it struck me as being appropriate. We were going back into a classroom setting and we were talking about school. And to have an avatar that reflected that, it was more interesting.
The desire for embodied avatars that can adapt to specific communication contexts was a common theme in the data. With respect to AMC in social VR, our results suggest that older adults are excited by the possibility of adapting their avatars to suit different virtual environments.

\subsection{Theme 3. Establishing social connections in AMC over distance}

A unique aspect and strength of our research is that it involved communication between groups of older adults, many of which had never met in real life and who were geographically separated by a distance of over $150 \mathrm{~km}$. This allowed us to examine how the AMC was influenced by people getting to know someone for the very first time and explore how communication over distance impacts on AMC in social VR. Thematic analysis of these exchanges revealed that participants invested time at the beginning of conversations probing the geographically distant group members in order to convince them they were really located where they said they were. Once this had been established, conversation focused on establishing shared connections.

Analysis of the video footage of the user study sessions showed that participants followed a similar process when meeting for the first time. The process consisted of two elements: polite conversation about socially acceptable topics but with an emphasis on location (typically, 'what is the weather like where you are?'), followed by specific questions about whether they had any shared interests or connections. This behaviour was remarkably consistent regardless of the composition of the group. In a typical example, after first discussing the weather, Yulia and Bernard [metropolitan] began questioning Wes [regional] about where he lived and where he went to school. This led to Bernard telling Wes that he had actually taught for a time in Wes' home town. Yulia was also able to establish that she knew a person who lived near Wes, and that she had spent some time as a young girl in a convent near Wes' home. When reflecting on this experience at the conclusion of the session, Wes spoke of its importance.

I thought [the AMC] was good quality, once we knew that...we all had a common interest...once you get started and people have an affinity with each other, it becomes very easy to communicate...[it] brought all three of us closer, you know, and we had no [reservations] about...discussing things, we were right into it.

It was interesting to note that when describing their experiences immediately after the social VR sessions, participants described meeting the person not interacting with an avatar. We took this to be a good sign that the connections made in the virtual environment were sufficiently immersive and realistic, a view supported the responses to the presence questionnaire as described in Table 2.

However, there were some examples where certain participants avatar appearance impacted negatively on other participants attempts to establish a strong social connection. Len, [a regional participant] expressed such feelings after a conversation with Cleo and Bernard. "[Cleo] said she looked similar to her avatar, I was trying to picture whether that was true or not?" Our analysis of the data identified several examples where participants expressed some 
confusion if another participant's description of themselves was not considered to be consistent with their avatar's appearance. As Herb, [a metropolitan participant] commented after a session with Klaus [regional]:

Sometimes I was wondering what [Klaus] really looked like. Sometimes the things he was saying didn't make sense, [his avatar] did not look like that... Because he was retired, [his avatar] could not have done that, you don't look like you could have done that.

The above results suggest there are specific social behaviours that are characteristic of geographically dispersed AMC in social VR. Our analysis suggests that while most participants felt high levels of presence during the social VR sessions, avatars add a further level of complexity to these interactions, especially when the users' conversation does not seem to align with the avatar's appearance.

\subsection{Theme 4. He had a giggle, but his face didn't giggle: Emotions and non-verbal cues in AMC}

Our final theme relates to how our participants reacted to our attempts to provide the avatars with more human-like expressions. A consistent theme of research with older adults examining AMC is that a lack of expressivity and behavioural anthropomorphism in avatars leads older adults to disassociate from them as a communication tool, often describing them as robots or puppets $[3,38]$. To attempt to address these issues, we implemented a number of features that aimed to improve behavioural anthropomorphism.

The first set of features combined the OVRLipSync plugin from Oculus to provide the ability for the avatar's lips to move when the user was speaking, and the Final IK plugin by Root Motion to simulate eye movement. Research into the use of simulated eye movement in social VR has been shown to result in "high quality of communication", even when compared to real eye movements captured with eye-tracking software [33, p.531].

Analysis of the user study data revealed that, with minor exceptions, these features were seen as being effective by our participants. When asked about their thoughts on their avatars, the majority of comments suggested that once the participant realised that their avatar was behaving appropriately, they spent little time thinking about it. Carl's comment was indicative of this view. "[Once I] saw who I was... I didn't feel the need to look at the [virtual mirror] again". Results from the questionnaire data bear this out. Of the 66 responses collected during the user study sessions, 37 responses (56.1\%) agreed or strongly agreed that the virtual environment seemed consistent with their real-world experiences, with only eight responses disagreeing or strongly disagreeing. Meanwhile 33 responses (50\%) agreed or strongly agreed with the statement 'it feels as if my avatar's body becomes my own', with only 13 responses disagreeing or strongly disagreeing.

A second set of features, however, was less successful. In order to allow participants some capacity to express a range of emotions, the prototype had a feature allowing them to adopt facial expressions by selecting preset emotions through pushing the Oculus hand controller's joystick in four separate directions. To familiarise the participants with the controls, they were asked to practice each control while looking at their avatar's face in the virtual mirror as part of the onboarding process. While some participants found it easy to manipulate the controls, others struggled with this interaction technique. The participants had the opportunity to give feedback on the usability of the prototype during its development (see Section 3), but the issues related to controlling their avatar's emotions only came to the fore during the social VR sessions.

Holly, in particular, found it difficult to remember which part of the controller to use, even after taking home a diagram of the controller's functionality to study in-between sessions. Analysis of the video data also revealed many instances where the flow of the conversation was interrupted because participants could not locate the appropriate emotion control on the hand controllers. Additionally, some participants - such as Ken, who had large hands - struggled to find a comfortable hand position on the controller and this led to further trouble trying to locate the emotion controls.

Moreover, though most participants could manage to manipulate the controls during the onboarding stage, they reported that it was not intuitive to use the controllers to express emotions when engaged in conversations with others. Bernard's comments are representative of many given in the post-session interviews: "You're concentrating on what you're trying to say...doing something with your thumb to control the emotions wasn't something I thought of." Len expressed his frustration that during one session he "had made a couple of comments and he [another participant] had a giggle, [but] his [avatar's] face didn't giggle." Analysis of the video footage of each session also revealed that on multiple occasions, participants accidentally pulled the wrong control, causing their avatar to express sadness or anger instead of smiling at their colleagues' avatars.

\section{DISCUSSION}

This paper reports on a five-month engagement with a group of older adults that aimed to better understand how older adults experience and view AMC in social VR. Our results, described within four complementary themes, show: how our participants compared AMC with other forms of communication; the impact that avatar appearance had on AMC; how AMC impacted on participants' attempts to establish social connections in social VR; and how our participants reacted to the prototype features that aimed to support their embodied avatars to express emotions and non-verbal cues. By adopting a participatory action research (PAR) methodology, we were able to partner with our participants throughout the project, including as co-authors on this paper, ensuring the trustworthiness of our results as an accurate reflection of their experience [16]. Our results respond to the question, How can older adult experiences of $A M C$ in social VR inform the design of social VR systems aimed at older users? In this section, we further discuss the results of our study and respond to the research question by offering six design reflections that speak to how the insights gained from the user study can be used to inform future design and research into older adults use of AMC in social VR.

\subsection{Comparing AMC with existing forms of communication}

The responses from our participants suggest that AMC in social VR is a communication medium that offers a number of compelling 
advantages that can benefit older adults. Our participants felt that AMC in social VR supported a level of anonymity that they appreciated and that this, in turn, supported the sharing of sensitive information and helped introverted users to express themselves more freely. They felt that AMC offered new opportunities to adapt avatar appearances so as to support communication in different circumstances and saw AMC in social VR as providing a good solution to the issue of divided attention that can impact on communication in other forms of computer mediated communication. However, AMC in social VR was also seem by some as being vastly inferior to face-to-face communication and both too complex and too abstracted when compared to their experiences using more mature computer mediated communication platforms such as FaceTime or Skype.

Anonymity has been shown to be particularly important to older adults with respect to computer-mediated communication [10]. Our results build on prior work that demonstrates AMC maintains the level of anonymity valued by older adults when communicating online [26, 38]. However, our work extends these earlier findings by offering new insights into how this aspect of AMC in social VR empowers and supports older people to discuss sensitive topics. As Yulia's example vividly demonstrates, our participants saw AMC in social VR as offering significant benefits over face-to-face and other forms of computer-mediated communication when discussing sensitive information. In highlighting this result, it is important to recognise that the School Days prototype was a closed social VR application. This allowed us to avoid many of the issues that impact on large open commercial social virtual worlds, which are subject to high levels of harassment [22].

Our results also emphasise the potential for AMC in social VR to be used as a tool to support shy and introverted older adults to engage in social conversation. As Holly's example demonstrates, some of the older adult participants in the user study felt they could contribute to group conversations more freely and openly via AMC in social VR. This further supports the view that AMC in social VR can be a powerful tool to support less confident people to (re)engage in social experiences. Personality traits such as introversion are positively associated with greater rates of depression in communitydwelling older adults [27], and depression is one of the greatest risk factors for social isolation [28]. Based on these results, we see an implication of our work being that older users see potential for AMC in social VR to be a tool that is appropriate for sharing a range of sensitive issues and can be a medium that can respond to the needs of a group of older adults who are at particular risk of social isolation, a topic that is of great interest to ageing researchers. Our first design reflections speak to these implications.

6.1.1 Design Reflection 1. Based on our participatory engagement with older adults during the user study, we believe that a closed social VR environment is an ideal platform in which older users can be supported to discuss sensitive topics. We believe that future design work that uses social VR and avatars to encourage older adults to discuss other positive and negative life experiences is an especially exciting opportunity, particularly if the interactions are facilitated by a trained counsellor or similar professional. We can see many opportunities to leverage this type of therapeutic social VR experience. For example, prior work has demonstrated that older adults who self-isolate in aged care settings are attracted to the idea of using VR to escape the confines of their environment [6]. We can envisage a range of AMC in social VR applications that can be used as therapeutic tools to connect older adults to supports beyond the confines of the age care setting so that they may share and support each other to address the many issues that have been shown to negatively impact on older adults in residential care $[14,48]$.

6.1.2 Design reflection 2. Our study suggests that AMC in social VR may be a communication medium that can support introverted users to express themselves more freely than they would be able to in face-to-face settings. Prior work has established that introverted people are at particular risk of social isolation [27, 28]. This is a significant finding given the long-standing interest within HCI on how technology can play a role in addressing the impacts of social isolation on older adults [4]. Based on our study, we believe that further empirical work is warranted in order to confirm these findings. One possible way forward could be to incorporate specific measurement of the benefits of AMC in social VR based on the 'Big Five' personality types [47].

6.1.3 Design reflection 3. Our results also demonstrate another potential advantage AMC in social VR has over videoconferencing technologies - namely that the immersive nature of AMC in social VR may solve the problem of divided attention. Based on our review of existing literature, we believe this is a topic that has been under-researched in HCI in this context [46]. As such, we would recommend that future $\mathrm{HCI}$ studies conduct comparative analysis of attention levels in both videoconferencing technology and AMC in Social VR in order to improve our understanding of this important factor.

\subsection{Adapting avatars to suit social circumstances}

The desire for embodied avatars that can adapt to specific social circumstances was another key theme in our data and we have already noted how this might support less confident users to engage in group conversation. A previous study of avatar use by older adults has shown that - particularly in relation to role playing games - older adults enjoy adapting avatars to a specific gaming context [11]. Our results extend this earlier work by showing that when communicating in social VR, our participants were excited by the possibilities they could envisage about how avatar appearances could be adapted to suit specific social contexts.

6.2.1 Design reflection 4. One design implication for this finding could be that future work might explore how avatars might be adapted to support the recall of previous memories. If successful, we could envisage a range of avatar design tools that could be developed to support AMC in social VR that targets older adults with mild cognitive impairment. There is anecdotal evidence that reminiscence in single-user VR can help older users to recollect forgotten memories [6]. We also see great opportunities for HCI researchers to design and evaluate social VR environments that specifically encourage other types of social interaction. For example, grandparents and grandchildren could share a bedtime story 
in social VR while embodying avatars of characters [and being immersed in a virtual environment modelled] from the book they are reading. Recent research examining the lessons that can be learned about how older adults can be supported as a result of the COVID-19 pandemic have emphasised the importance of leveraging technology for this type of inter-generational support [23].

6.2.2 Design reflection 5. While the finding that participants were attracted to the idea of adapting avatars to suit social contexts has clear implications for creative future design work as described above, we also believe that a note of caution is warranted. While Samuel's attempt to try and embody a female avatar was motivated by a genuine curiosity and desire to 'better fit in' during conversations with women participants, the ability to deliberately misrepresent oneself in social VR for nefarious purposes cannot be discounted. Prior work has noted the complex social challenges that accompanied the decision to introduce a user's voice into virtual worlds such as Second Life [44]. Therefore, we would also recommend that future work investigates strategies for improving user trust of AMC in social VR in order to prevent similar challenges in future social VR applications.

\subsection{Emotions and non-verbal communication in AMC}

A lack of behavioural anthropomorphism has been a commonly cited issue with AMC that has often resulted in older adults feeling a lack of social presence that causes them to mistrust - or simply not enjoy - AMC [38]. A lack of non-verbal cues has also resulted in older adults criticising avatars as being too robotic or doll-like to support genuine communication [3,38]. In earlier stages of our project, our participants had echoed many of these concerns and this led to design decisions being made to improve the ability of the social VR prototype to facilitate emotional expression and non-verbal cues. Results from the user study demonstrate that by incorporating a range of technologies that allow for capturing, or inferring, facial expressions and non-verbal cues, many of these concerns were alleviated. Based on these results, we believe that future design work on AMC in social VR will benefit from incorporating and building on our implementation of specific methods to improve avatar anthropomorphism.

6.3.1 Design reflection 6. While our incorporation of measures to improve the behavioural anthropomorphism of the avatars in the user study were broadly successful, results also highlight some areas where there remains significant room for improvement. In particular, our attempts to allow for emotion control via the Oculus' dedicated hand controls resulted in a poor user experience for our participants. This suggests that any kind of multi-tasking or manual requirements associated with expressing emotions is likely to be rejected by older social VR users. Ideally, emotions would be triggered automatically. We note the body of work that continues to investigate automated ways to incorporate emotional expressions onto avatars in virtual environments [7, 15, 31]. Our research contributes to these ongoing efforts by stressing that any successful emotion inferring system must be robust enough that non-technical users can use the feature without the need for intrusive, fragile or expensive equipment. Moreover, our results also highlight the need for future research that carefully considers whether and when older users would like to share or hide their emotions in social VR.

\subsection{Older adults contributing to the design of future social VR systems}

In addition to the design reflections outlined above, our study contributes to the growing conversation in HCI about the value of engaging older adults in participatory research. Our participatory action research project, spanning over three years in total, has provided an important illustration of how older adults can play an active role in evaluating and improving the design of technologies that target older users. In reviewing the data collected during the user study, we were struck by our participants' ability to draw on their life experience to contextualise their thoughts on AMC. We believe this is a particular strength of engaging older adults in technology evaluations and argue that this benefit could extend beyond designing technologies specifically targeting older users. As one of our participants, Len, put it during the participatory design work that preceded the user study, older adults bring a unique perspective to technology evaluation in that they are "coming from a different point of discovery". We wholeheartedly endorse this view and hope that another contribution of our study can be its ability to act as a catalyst for more participatory engagement with older adults across all domains of $\mathrm{HCI}$ research.

\section{LIMITATIONS AND FUTURE WORK}

Our data is based on participants' responses to one social VR prototype. During their reflections, participants introduced the topic of video-mediated communication and had a tendency to compare this medium with social VR. Since the present study was not designed to explore this difference in detail, future research could perform a comparison between social VR, video-mediated communication and other technologies to explore the perceived advantages and drawbacks in a more systematic way. An additional area for future work is to explore the use of social VR 'in the wild'. Like the majority of published studies on AMC and older adults (e.g. [38]), the present study was lab-based. Future studies in older adults' homes or even aged care settings can extend our understanding of how AMC in social VR should be designed to improve older adults' lives.

\section{CONCLUSION}

In this paper we have demonstrated that AMC in social VR provides a promising avenue for supporting social connectedness in later life. Through our analysis of data collected during a five-month user study with 16 participants aged 70 to 81 , we identified how AMC in social VR offers advantages over face-to-face communication when discussing sensitive topics. Our results demonstrate how shy and introverted older adults see AMC in social VR as a more comfortable environment for them to communicate, a finding that supports the view that future social VR systems have a strong role to play in supporting socially isolated older adults to (re)connect with social supports. Our participants were excited about the potential to adapt their embodied avatars for specific communication roles and our analysis reveals how this opens up design possibilities for social VR systems that allow those with mild cognitive impairment to recollect forgotten memories. Our five-month engagement with our 
participants also allowed us to evaluate lip syncing and inferred eye movement plugins that aimed to improve the ability of embodied avatars to facilitate emotional expression and non-verbal ques. Our user study demonstrates the advantage of providing older adults with an active role in evaluating technologies that are aimed at older users and provides a template for the increased involvement of older adults in future $\mathrm{HCI}$ research.

\section{ACKNOWLEDGMENTS}

This work is supported by an ARC Discovery grant (Project ID DP160101368). The first author would like to thank Zaher Joukhadar and Martin Reinoso for additional technical support during the study. We also thank the anonymous reviewers for suggestions that improved this paper. Finally, we would like to thank all the participants who took part in the study for their time and contributions to the project.

\section{REFERENCES}

[1] Jeremy N Bailenson, Nick Yee, Jim Blascovich, and Rosanna E Guadagno. 2008. Transformed social interaction in mediated interpersonal communication. In Mediated Interpersonal Communication, Elly A Konijn, Sonja Utz, Martin Tanis, and Susan B Barnes (Eds.). Routledge.

[2] Steven Baker, Ryan M. Kelly, Jenny Waycott, Romina Carrasco, Roger Bell, Zaher Joukhadar, Thuong Hoang, Elizabeth Ozanne, and Frank Vetere. 2020. School's Back: Scaffolding Reminiscence in Social Virtual Reality with Older Adults. Proceedings of the ACM on Human-Computer Interaction 4, CSCW, Article 267 (Dec. 2020), 25 pages. https://doi.org/10.1145/3434176

[3] Steven Baker, Ryan M. Kelly, Jenny Waycott, Romina Carrasco, Thuong Hoang, Frances Batchelor, Elizabeth Ozanne, Briony Dow, Jennifer Warburton, and Frank Vetere. 2019. Interrogating Social Virtual Reality as a Communication Medium for Older Adults. Proceedings of the ACM on Human-Computer Interaction 3, CSCW, Article 149 (Nov. 2019), 24 pages. https://doi.org/10.1145/3274356

[4] Steven Baker, Jeni Warburton, Jenny Waycott, Frances Batchelor, Thuong Hoang, Briony Dow, Elizabeth Ozanne, and Frank Vetere. 2018. Combatting social isolation and increasing social participation of older adults through the use of technology: A systematic review of existing evidence. Australasian fournal on Ageing 37, 3 (Sept. 2018), 184-193.

[5] Steven Baker, Jenny Waycott, Romina Carrasco, Thuong Hoang, and Frank Vetere. 2019. Exploring the Design of Social VR Experiences with Older Adults. In Proceedings of the 2019 on Designing Interactive Systems Conference - DIS '19 (San Diego, CA, USA) (DIS '19). ACM Press, New York, New York, USA, 303-315.

[6] Steven Baker, Jenny Waycott, Elena Robertson, Romina Carrasco, Barbara Barbosa Neves, Ralph Hampson, and Frank Vetere. 2020. Evaluating the use of interactive virtual reality technology with older adults living in residential aged care. Inf. Process. Manag. 57, 3 (May 2020), 102105.

[7] Guillermo Bernal and Pattie Maes. 2017. Emotional Beasts: Visually Expressing Emotions through Avatars in VR. In Proceedings of the 2017 CHI Conference Extended Abstracts on Human Factors in Computing Systems (Denver, Colorado, USA) (CHI EA '17). Association for Computing Machinery, New York, NY, USA, 2395-2402.

[8] Virginia Braun and Victoria Clarke. 2006. Using thematic analysis in psychology. Qual. Res. Psychol. 3, 2 (Jan. 2006), 77-101.

[9] Virginia Braun and Victoria Clarke. 2012. Thematic analysis. In APA handbook of research methods in psychology, Vol 2: Research designs: Quantitative, qualitative, neuropsychological, and biological., Harris Cooper, Paul M Camic, Debra L Long, A T Panter, David Rindskopf, and Kenneth J Sher (Eds.). American Psychological Association, Washington, 57-71.

[10] Robin Brewer and Anne Marie Piper. 2016. Tell It Like It Really Is: A Case of Online Content Creation and Sharing Among Older Adult Bloggers. In Proceedings of the 2016 CHI Conference on Human Factors in Computing Systems. ACM, New York, NY, 5529-5542.

[11] Romina Carrasco, Jenny Waycott, Steven Baker, and Frank Vetere. 2018. Designing the Lost Self: Older Adults' Self-representations in Online Games. In Proceedings of the 2018 Designing Interactive Systems Conference (Hong Kong, China) (DIS '18). ACM, New York, NY, USA, 441-452.

[12] Annie T Chen, Shaoqing Ge, Susie Cho, Andrew K Teng, Frances Chu, George Demiris, and Oleg Zaslavsky. 2020. Reactions to COVID-19, information and technology use, and social connectedness among older adults with pre-frailty and frailty. Geriatr. Nurs. (Aug. 2020).

[13] Nicole Cook and Sandra L Winkler. 2016. Acceptance, Usability and Health Applications of Virtual Worlds by Older Adults: A Feasibility Study. FMIR Res.
Protoc. 5, 2 (June 2016), e81.

[14] Linél Franck, Natalie Molyneux, and Lynne Parkinson. 2016. Systematic review of interventions addressing social isolation and depression in aged care clients. Oual. Life Res. 25, 6 (June 2016), 1395-1407.

[15] J D Hart, T Piumsomboon, L Lawrence, G A Lee, and others. 2018. Emotion sharing and augmentation in cooperative virtual reality games. Proceedings of the (2018).

[16] Gillian R Hayes. 2011. The relationship of action research to human-computer interaction. ACM Trans. Comput. Hum. Interact. 18, 3 (2011), 1-20.

[17] Jason Hayhurst. 2018. How Augmented Reality and Virtual Reality is Being Used to Support People Living with Dementia-Design Challenges and Future Directions. In Augmented Reality and Virtual Reality: Empowering Human, Place and Business, Timothy Jung and M Claudia tom Dieck (Eds.). Springer International Publishing, Cham, 295-305.

[18] Aram Kim, Nora Darakjian, and James M Finley. 2017. Walking in fully immersive virtual environments: an evaluation of potential adverse effects in older adults and individuals with Parkinson's disease. Journal of NeuroEngineering and Rehabilitation 14, 1 (Feb. 2017), 16.

[19] Valentina La Corte, Marco Sperduti, Kouloud Abichou, and Pascale Piolino. 2019. Episodic Memory Assessment and Remediation in Normal and Pathological Aging Using Virtual Reality: A Mini Review. Front. Psychol. 10 (Feb. 2019), 173.

[20] B S Lange, P Requejo, S M Flynn, A A Rizzo, F J Valero-Cuevas, L Baker, and C Winstein. 2010. The potential of virtual reality and gaming to assist successful aging with disability. Phys. Med. Rehabil. Clin. N. Am. 21, 2 (May 2010), 339-356.

[21] Jie Li, Yiping Kong, Thomas Röggla, Francesca De Simone, Swamy Ananthanarayan, Huib de Ridder, Abdallah El Ali, and Pablo Cesar. 2019. Measuring and Understanding Photo Sharing Experiences in Social Virtual Reality. In Proceedings of the 2019 CHI Conference on Human Factors in Computing Systems (Glasgow, Scotland Uk) (CHI '19, Paper 667). Association for Computing Machinery, New York, NY, USA, 1-14.

[22] Joshua McVeigh-Schultz, Anya Kolesnichenko, and Katherine Isbister. 2019. Shaping Pro-Social Interaction in VR: An Emerging Design Framework. In Proceedings of the 2019 CHI Conference on Human Factors in Computing Systems (Glasgow, Scotland Uk) (CHI '19). Association for Computing Machinery, New York, NY, USA, 1-12. https://doi.org/10.1145/3290605.3300794

[23] Nancy Morrow-Howell, Natalie Galucia, and Emma Swinford. 2020. Recovering from the COVID-19 Pandemic: A Focus on Older Adults. F. Aging Soc. Policy 32, 4-5 (July 2020), 526-535.

[24] William Lawrence Neuman. 2011. Social research methods : qualitative and quantitative approaches. Allyn \& Bacon, Boston.

[25] Kristine Nowak and Jesse Fox. 2018. Avatars and computer-mediated communication: a review of the definitions, uses, and effects of digital representations. Review of Communication Research 6 (2018), 30-53.

[26] Catherine J O'Brien, Jennifer L Smith, and Dennis E Beck. 2016. Real relationships in a virtual world: Social engagement among older adults in Second Life. Gerontechnology 15 (2016), 171-179.

[27] D M O'Shea, V M Dotson, and R A Fieo. 2017. Aging perceptions and self-efficacy mediate the association between personality traits and depressive symptoms in older adults. Int. f. Geriatr. Psychiatry 32, 12 (Dec. 2017), 1217-1225.

[28] Andrea Poscia, Jovana Stojanovic, Daniele Ignazio La Milia, Mariusz Duplaga, Marcin Grysztar, Umberto Moscato, Graziano Onder, Agnese Collamati, Walter Ricciardi, and Nicola Magnavita. 2018. Interventions targeting loneliness and social isolation among the older people: An update systematic review. Exp. Gerontol. 102 (Feb. 2018), 133-144.

[29] Peter Reason and Hilary Bradbury. 2008. The Sage handbook of action research : participative inquiry and practice. SAGE Publications, Thousand Oaks, California.

[30] Darren J Reed and Geraldine Fitzpatrick. 2008. Acting Your Age in Second Life. In Lecture Notes in Computer Science. 158-169.

[31] Jonas Reichenberger, Michael Pfaller, and Andreas Mühlberger. 2020. Gaze Behavior in Social Fear Conditioning: An Eye-Tracking Study in Virtual Reality. Front. Psychol. 11 (Jan. 2020), 35.

[32] Amy Restorick Roberts, Bob De Schutter, Kelley Franks, and M Elise Radina. 2019. Older Adults' Experiences with Audiovisual Virtual Reality: Perceived Usefulness and Other Factors Influencing Technology Acceptance. Clin. Gerontol. 42, 1 (Jan. 2019), 27-33

[33] Sven Seele, Sebastian Misztal, and Helmut Buhler. 2017. Here's Looking At You Anyway! How Important is Realistic Gaze Behavior in Co-located Social Virtual Reality Games?. In CHI PLAY 2017 (Amsterdam, NL).

[34] Alexander Seifert. 2020. The Digital Exclusion of Older Adults during the COVID19 Pandemic. 7. Gerontol. Soc. Work (May 2020), 1-3.

[35] Sara Shaunfield, Elaine Wittenberg-Lyles, Debra Parker Oliver, and George Demiris. 2014. Virtual Field Trips for Long-Term Care Residents: A Feasibility Study. null 38, 3 (July 2014), 237-247.

[36] Panote Siriaraya and Chee Siang Ang. 2012. Characteristics and usage patterns of older people in a 3D online multi-user virtual environment. Comput. Human Behav. 28, 5 (Sept. 2012), 1873-1882.

[37] Panote Siriaraya and Chee Siang Ang. 2014. Recreating living experiences from past memories through virtual worlds for people with dementia. In Proceedings of 
the SIGCHI Conference on Human Factors in Computing Systems. ACM, 3977-3986.

[38] Panote Siriaraya and Chee Siang Ang. 2019. The Social Interaction Experiences of Older People in a 3D Virtual Environment. In Perspectives on Human-Computer Interaction Research with Older People, Sergio Sayago (Ed.). Springer International Publishing, Cham, 101-117.

[39] Panote Siriaraya, Chee Siang Ang, and Ania Bobrowicz. 2014. Exploring the potential of virtual worlds in engaging older people and supporting healthy aging Behav. Inf. Technol. 33, 3 (March 2014), 283-294.

[40] Panote Siriaraya and Chee Siang Ang. 2012. Age differences in the perception of social presence in the use of $3 \mathrm{D}$ virtual world for social interactionq. Interact. Comput. 24, 4 (July 2012), 280-291.

[41] Nina Skjæret, Ather Nawaz, Tobias Morat, Daniel Schoene, Jorunn Lægdheim Helbostad, and Beatrix Vereijken. 2016. Exercise and rehabilitation delivered through exergames in older adults: An integrative review of technologies, safety and efficacy. Int. 7. Med. Inform. 85, 1 (Jan. 2016), 1-16.

[42] Misha Sra, Aske Mottelson, and Pattie Maes. 2018. Your Place and Mine: Designing a Shared VR Experience for Remotely Located Users. In Proceedings of the 2018 Designing Interactive Systems Conference (Hong Kong, China) (DIS '18). ACM, New York, NY, USA, 85-97.
[43] Cosimo Tuena, Elisa Pedroli, Pietro Davide Trimarchi, Alessia Gallucci, Mattia Chiappini, Karine Goulene, Andrea Gaggioli, Giuseppe Riva, Fabrizia Lattanzio, Fabrizio Giunco, and Marco Stramba-Badiale. 2020. Usability Issues of Clinical and Research Applications of Virtual Reality in Older People: A Systematic Review. Front. Hum. Neurosci. 14 (April 2020), 93.

[44] Greg Wadley and Martin R Gibbs. 2010. Speaking in character: Voice communication in virtual worlds. In Online worlds: Convergence of the real and the virtual. Springer, $187-200$.

[45] Bob G Witmer and Michael J Singer. 1998. Measuring Presence in Virtual Environments: A Presence Questionnaire. Presence: Teleoperators and Virtual Environments 7, 3 (June 1998), 225-240.

[46] Xiang Xiao and Jingtao Wang. 2017. Undertanding and Detecting Divided Attention in Mobile MOOC Learning. In Proceedings of the 2017 CHI Conference on Human Factors in Computing Systems. ACM, 2411-2415.

[47] Nick Yee, Helen Harris, Maria Jabon, and Jeremy N Bailenson. 2011. The Expression of Personality in Virtual Worlds. Soc. Psychol. Personal. Sci. 2, 1 (Jan. 2011), 5-12.

[48] Gabriella Zizzo, Catherine Mackenzie, Carol Irizarry, and Ian Goodwin-Smith. 2020. Loss and grief: The experience of transition to residential aged care. Aust. 7. Soc. Issues 55, 4 (Dec. 2020), 474-491. 


\section{University Library}

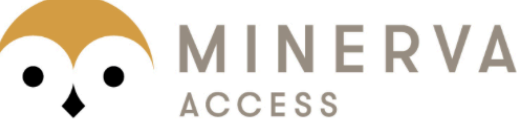

A gateway to Melbourne's research publications

Minerva Access is the Institutional Repository of The University of Melbourne

Author/s:

Baker, S;Waycott, J;Carrasco, R;Kelly, R;Jones, A;Lilley, J;Dow, B;Batchelor, F;Hoang,

T; Vetere, F

Title:

Avatar-Mediated Communication in Social VR: An In-depth Exploration of Older Adult Interaction in an Emerging Communication Platform

Date:

2021-05-08

Citation:

Baker, S., Waycott, J., Carrasco, R., Kelly, R., Jones, A., Lilley, J., Dow, B., Batchelor, F., Hoang, T. \& Vetere, F. (2021). Avatar-Mediated Communication in Social VR: An In-depth Exploration of Older Adult Interaction in an Emerging Communication Platform. Proceedings of the 2021 ACM CHI Conference on Human Factors in Computing Systems., pp.1-13. Association for Computing Machinery (ACM). https://doi.org/10.1145/3411764.3445752.

Persistent Link:

http://hdl.handle.net/11343/258786

License:

Publisher's own licence 